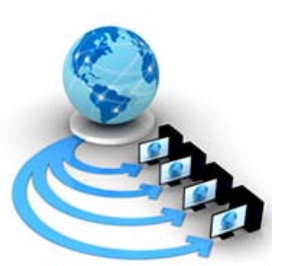

Volume 8, No. 9, November-December 2017

International Journal of Advanced Research in Computer Science

RESEARCH PAPER

Available Online at www.ijarcs.info

\title{
DETECTING USER LINKAGE ACROSS DIFFERENT SOCIAL MEDIA PLATFORMS THROUGH MULTI-OBJECTIVE LEARNING FRAMEWORK
}

\author{
Konatham NagaSwetha \\ M. Tech Student, Computer Science \& Engineering \\ Anurag Group of institutions \\ Village Venkatapur, Mandal Ghatkesar, District \\ RangaReddy, Telangana, India
}

\author{
Sudeepthi \\ Assistant Professor, Computer Science \& Engineering \\ Anurag Group of institutions \\ Village venkatapur, Mandal Ghatkesar, District \\ RangaReddy, Telangana, India
}

\begin{abstract}
In this day and an individual people can make utilization of various online networking stages for various purposes. Regularly the data gave on the individual set is not finished as well as divided. Linkage of character crosswise over online networking increases further comprehension of client profiles. More profound comprehension of client information mostly helps in business insight. This paper contains a system called HYDRA which comprises of 3 stages: (i) demonstrate the heterogeneous behaviour of client (ii) fabricate the structure consistency design (iii) optimization.
\end{abstract}

Keywords: Heterogeneous behaviour, Structure learning, Social Media Platforms

\section{INTRODUCTION}

The capacity of accepting different personalities has for quite some time been a fantasy for some individuals. However it is not until the late appearance of online informal communities that this aspiration of millions has been made conceivable in digital virtual world [1], [2], [3], [5], [9]. Truth be told, the current expansion of interpersonal organization administrations of various types has changed our social life by giving everybody the simplicity and fun of sharing distinctive data more than ever (e.g., small scale online journals, pictures, recordings, audits, and area checkins). In the mean time, likely the greatest and most fascinating inquiry concerning all organizations is the manner by which to use this huge social information for better business knowledge. Specifically, individuals think about how to increase careful comprehension of every individual client from the huge measure of online social information records [4]. Tragically, data of a client from the present social scene is divided, conflicting and troublesome. The way to releasing the genuine energy of webbased social networking is to connect up every one of the information of a similar client crosswise over various social stages, offering the accompanying advantages to client profiling.

Individuals have accounts in various interpersonal organization stages like Face book, Twitter, and Orkut and so forth. They keep up their record under various characters. It's exceptionally hard to locate the single client personality among these system stages. Since, in digital system additionally no one has seen these deceitful exercises. Presently a day, everybody shares their data through informal network administrations. In the meantime, the greatest inquiry emerges is the way to get these clients huge information for distinguishing the genuine client. So individuals were thinking how to get that individual client data from different stages. In any case, because of same individual keeping up accounts in various online networking stages, utilizing the logs of one stage alone to get the client conduct is not a decent practice. So there ought to be an approach to interface the distinctive online networking records to single client and gather every one of the records for that client from all stages. At exactly that point revise client conduct and interests has been derived. In this task, we give an answer for connecting distinctive records in various web-based social networking stages to a solitary client. This can give the different advantages like cross checking the client character would empowers us to careful comprehension of an every individual client's data and the structure of their conduct designs.

\section{RELATED WORK}

Client linkage was initially formalized as interfacing relating characters crosswise over groups and a web-look based approach was proposed to address it [1]. Past research can be classified into three sorts: client profile-based, client created content based and client conduct display based. Client profile-based techniques gather labeling data gave by clients or client profiles from a few interpersonal organizations and after that speak to client profiles in vectors, of which each measurement relates to a profile field (e.g., username, profile picture, portrayal, area, occupation, and so on.). Existing techniques in this class experience the ill effects of immense exertion of client labeling, distinctive identifiable individual data sorts from site to site, and security of client profile. [7] Client created content-based strategies, then again, gather individual identifiable data from open pages of client produced content. However these techniques still make the supposition of predictable usernames crosswise over social stages, which is not the situation in huge scale interpersonal organization stages. Client conduct display based strategies investigate conduct examples and assemble highlight models from usernames, dialect and composing styles. Tragically, past techniques 1) have not dealt with the missing data common among usernames, client produced content, client conduct and social structures; 2) have not investigated the fundamental explanations behind the missing data and its effect on client character linkage; 3) have not very much formalized the client linkage issue with an answer of a sound hypothetical establishment. To the best of our insight, our work is the first to connect clients crosswise over various online networking stages by coordinating all the social information related with a client in a brought together model.

O. Hassanzadeh, K. Q. Pu, S. H. Yeganeh, R. J. Mill operator, M. Hernandez, L. Popa, and H. Ho [6], exhibited a system for finding linkage focuses over expansive semi organized Web information. Our system incorporates a library of lexical analyzers, a library of similitude works, and an arrangement of novel inquiry calculations alongside viable separating procedures. We tentatively assessed our structure in nine situations including genuine Web information sources in three spaces. The informational collections utilized as a part of our trials alongside the outcomes are accessible. We are as of now investigating various intriguing bearings for future work. We are intending to additionally stretch out our comparability work library to incorporate capacities that consider semantic closeness of the qualities notwithstanding syntactic and lexical coordinating. We will 
probably stretch out our framework to distribute the linkage focuses we find utilizing existing benchmarks, for example, the as of late proposed R2R mapping dialect.

Reza Zafarani et al [3], [9] examined the client linkage crosswise over differed groups and upheld the online pursuit approach things being what they are usernames over the different groups is distinguished and furthermore the relating usernames in different groups are considered. T. Iofciu et al [10] examine whether clients is distinguished crosswise over social labeling frameworks and gather the client labeling information for identification the specific client and these client profiles is shown in vector sort.

$\mathrm{N}$. Korula et al [12] creators planned the local dispersion calculation for interpersonal organization compromise to connect the monstrous arrangement of client recognizable proof hubs in two diverse system diagrams. For this reason just the basic subtle elements is utilized. R. Zheng et al anticipated a structure for recognizing the approval of the client and messages sent by the client through on the web. This structure will receive the accentuations used in the content and semantic choices and so forth and it moreover join the capacities used in the words. A. Nunes et al [11] portrays an approach for determination the client personality issues by contrasting the changed alternatives and traits of the client. This will order the client data in paired kind to discover regardless of whether the information has a place with same. By consolidating this client or not highlight vectors, the closeness score is ascertained. For instance, closeness between client essential information like name, sex, age, date of birth and client went by area and so on.

J. Liu et al [2] anticipated semi administered learning system to delineate clients crosswise over informal organizations and hence a similar client profiles inside the diverse stages to look at for the similitude has been induced. For each system the diagram is drawn and later it fabricates the semi administered structure to check between the two clients.

\section{FRAMEWORK}

\section{A. Proposed System Overview}

In this paper, we have a tendency to propose HYDRA that coordinates each client's heterogeneous behavior as well as their center interpersonal organization structure into an assembled multitarget client linkage system. HYDRA comprises of the accompanying three primary strides.

Step1. Behavior Similarity Modeling We figures the comparability between a couple of clients of fight all client sets through heterogeneous conduct displaying.

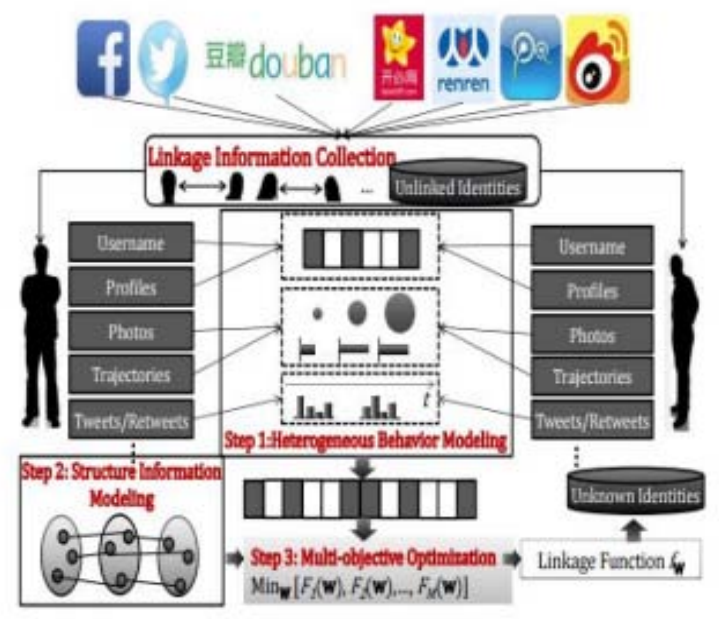

Fig1. Proposed HYDRA Framework

Step2: Structure Consistency Modeling: We build the structure consistency chart on client matches by considering each the center system structure of the clients and their behavior likenesses.

Step3: Multi-objective optimization [8]: Supported the past a couple of steps, we tend to change over the SIL hindrance into a two-class arrangement burden and develop multi target change that together improves the figuring exactness on the named client sets and various structure consistency measurement crosswise over totally unique stages.

\section{B. A framework of authorship identification of online messages}

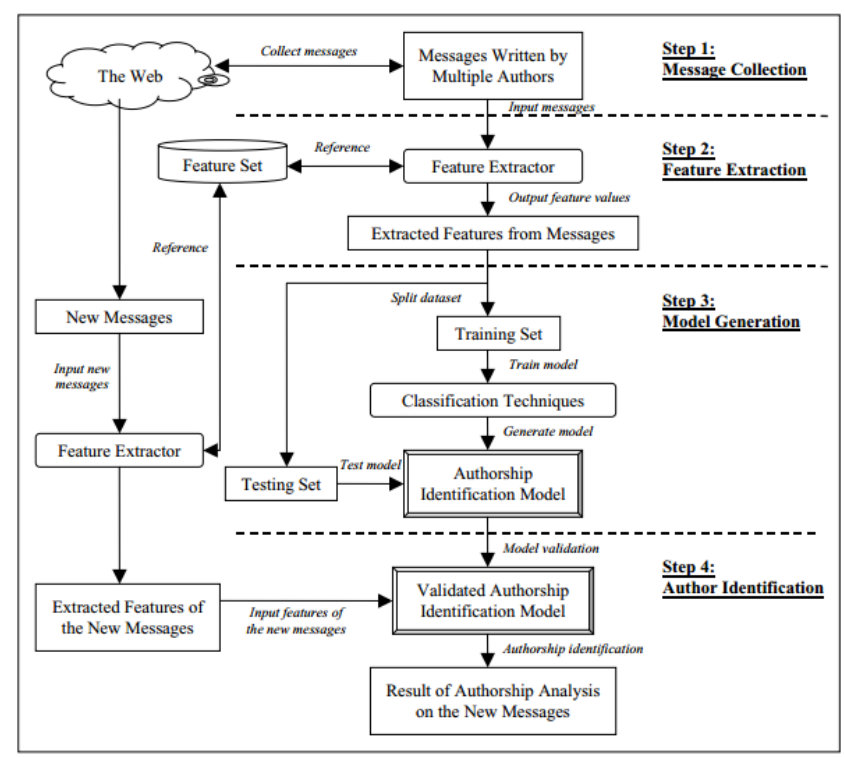

Fig. 2 a framework of authorship identification of online messages

\section{Message collection}

Based on previous investigations on the online, investigators need to collect a group of on-line messages written by promising authors to profile the writing forms of every author.

\section{Feature Extraction}

Online messages on the web are in unstructured text format. Supported the predefined writing-style options, the feature extractor will analyze the messages and extract the options in matter on-line messages. Once feature extraction, every unstructured text is painted as a vector of writing style options.

\section{Model Generation}

As in a very typical classifier learning method, the online message collection is split into 2 subsets [1]. One subset, known as the coaching set, is employed to train the classification model. The classification techniques applied during this method could cause models with totally different predictive powers. The opposite set is named the testing set that is employed to validate the prediction power of the authorship-identification model generated by the classification model. If the performance of the classifier is verified by the testing set, it is often wont to establish the authorship of freshly found on-line messages. A reiterative coaching and testing method may be required to develop a good authorship-prediction model.

\section{Author Identification}

After the authorship-identification model is developed; it are often used to predict the authorship of unknown on-line messages. The results of authorship identification can facilitate the investigator focus his or her effort on a small set of messages and authors.

\section{EXPERIMENTAL RESULTS}

In our experiments, we take two datasets which are belongs to social media. After upload two datasets, we need to build the vectors for uploaded dataset. 


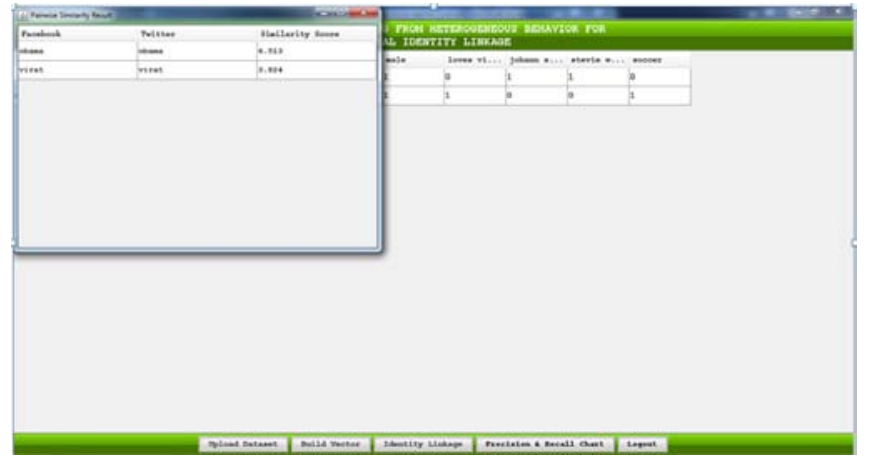

Here, we are detecting the similar user in two types of dataset. For that reason we find the pair wise similarity between all users in the dataset.

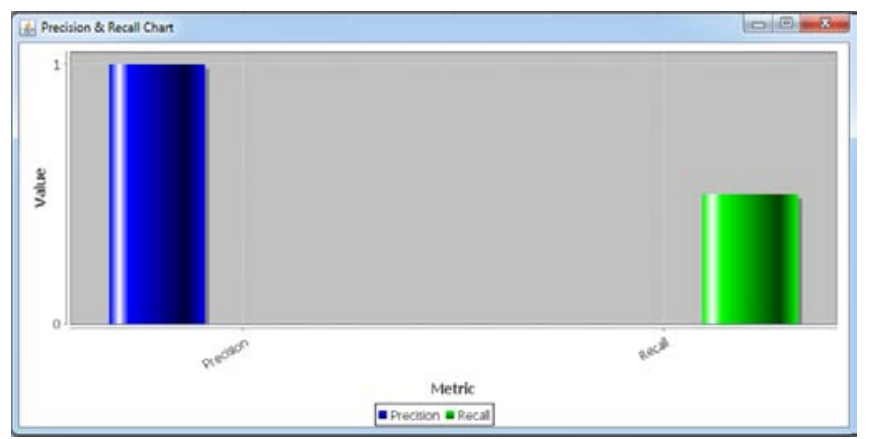

From our experiment, we proved that the proposed HYDRA framework is better than the traditional approaches to detect the social identity linkage across social media platforms.

\section{CONCLUSION}

In this project, we conclude that we proposed a frame work HYDRA. Through this framework we solved the user identity linkage on the social media platforms. In this project we propose a heterogeneous behavior model to calculate the user behavior similarity from user's social data. And in this project we propose structure modeling method to improve the behavior consistency on user core instead of behavior similarity. Through the experimental results, we can say that our system is better than the traditional systems to find user identity linkage on the social media platforms.

\section{REFERENCES}

[1] Siyuan Liu, Shuhui Wang, Feida Zhu "Structured Learning from Heterogeneous Behavior for Social Identity Linkage", IEEE Transactions On Knowledge Discovery And Engineering Vol No 27 Year 2015

[2] J. Liu, F. Zhang, X. Song, Y.-I. Song, C.-Y. Lin, and H.-W. Hon, "What's in a name?: an unsupervised approach to link users across communities," in WSDM'13, 2013.

[3] R. Zafarani and H. Liu, "Connecting users across social media sites: A behavioral-modeling approach,” in KDD’13, 2013.

[4] S. Kumar, R. Zafarani, and H. Liu, "Understanding user migration patterns in social media,” in AAAI'11, 2011, pp. $-1-1$.

[5] R. Zheng, J. Li, H. Chen, and Z. Huang, “A framework for authorship identification of online messages: Writing-style features and classification techniques," Journal of the Association for Information Science and Technology, vol. 57, no. 3, 2006.

[6] O. Hassanzadeh, K. Q. Pu, S. H. Yeganeh, R. J. Miller, M. Hernandez, L. Popa, and H. Ho, "Discovering linkage points over web data,” PVLDB, vol. 6, no. 6, pp. 444-456, 2013.

[7] G. Pickard, W. Pan, I. Rahwan, M. Cebrian, R. Crane, A. Madan, and A. Pentland, "Time-critical social mobilization,” Science, vol. 334, no. 6055, pp. 509-512, 2011.

[8] R. T. Marler and J. S. Arora, "Survey of multi-objective optimization methods for engineering," Structural and multidisciplinary optimization, vol. 26, no. 6, pp. 369-395, 2004

[9] R. Zafarani and H. Liu, "Connecting corresponding identities across communities,” in ICWSM'09, 2009, pp. $1-1$.

[10] T. Iofciu, P. Fankhauser, F. Abel, and K. Bischoff, "Identifying users across social tagging systems," in ICWSM'11, 2011, pp. -1-1

[11] A. Nunes, P. Calado, and B. Martins, "Resolving user identities over social networks through supervised learning and rich similarity features," in SAC'12, 2012, pp. 728729.

[12] N. Korula and S. Lattanzi, "An efficient reconciliation algorithm for social networks,” PVLDB, pp. 377-388, 2014. 